\title{
Prolongements en fonctions algébriquement constructibles
}

\author{
Isabelle BONNARD-DORÉ \\ Lycée Poincaré, \\ 2 rue de la Visitation, BP 4202, \\ 54042 Nancy Cedex, France \\ isabelle.bonnard@laposte.net
}

Recibido: 7 de Abril de 2003

Aceptado: 5 de Mayo de 2004

\begin{abstract}
In this paper we consider the following question: Let $S$ be a semialgebraic subset of a real algebraic set $V$, and let $\varphi: S \rightarrow \mathbb{Z}$ be a function on $S$. Is $\varphi$ the restriction of an algebraically constructible function on $V$, i.e. a sum of signs of polynomials on $V$ ? We give an effective method to answer this question when $\varphi(S) \subset\{-1,1\}$ or $\operatorname{dim} S \leq 2$ or $S$ is basic.
\end{abstract}

Key words: semialgebraic sets, algebraically constructible functions, quadratic forms.

2000 Mathematics Subject Classification: 14P10

\section{Introduction}

Soit $V \subset \mathbb{R}^{N}$ un ensemble algébrique réel. (Dans tout cet article, les ensembles algébriques considérés sont des zéros de polynômes dans $\left.\mathbb{R}^{N}\right)$. Les fonctions algébriquement constructibles sur $V$ ont été introduites par C. McCrory et A. Parusiński pour étudier la topologie des ensembles algébriques réels. (Voir [10] pour les dimensions $\leq 3$, et [11] pour la dimension 4). Elles sont définies comme les combinaisons linéaires, à coefficients entiers, de caractéristique d'Euler-Poincaré de morphismes polynômiaux propres (cf. [10]). Par [9] ou [13], ces fonctions coïncident avec les sommes de signes de polynômes sur $V$, où le signe d'un polynôme $P$ sur $V$ est la fonction $\operatorname{sgn} P$ : $V \rightarrow\{-1,0,1\}$ qui vaut 1 (respectivement $-1,0$ ) là où $P$ est strictement positif (respectivement strictement négatif, nul). 
On s'intéresse au problème suivant. Soit $S$ un sous-ensemble semi-algébrique de $V$ et soit $\varphi: S \rightarrow \mathbb{Z}$ une fonction constructible, i.e. telle qu'il existe une partition semialgébrique finie de $S$ sur chaque élément de laquelle $\varphi$ est constante. La fonction $\varphi$ est-elle la restriction à $S$ d'une fonction algébriquement constructible sur $V$ tout entier?

Nous montrons comment répondre de manière effective à cette question lorsque $\varphi$ ne prend que les valeurs -1 et 1 , lorsque la dimension de $S$ est au plus deux, ou lorsque le semi-algébrique $S$ est de dimension plus grande mais basique. (On dit qu'un semialgébrique est basique s'il est donné par une conjonction d'inégalités polynomiales).

Pour cela, nous utilisons le spectre réel de l'anneau des polynômes sur $V$, et les formes quadratiques sur ce spectre réel. En effet, les signatures de ces formes quadratiques sont en correspondance avec les fonctions algébriquement constructibles.

Le plan de l'article est le suivant. Nous commencerons par des rappels sur le spectre réel. Dans la deuxième section, nous montrerons comment nous ramener à travailler génériquement, i.e. à un sous-ensemble algébrique de dimension strictement inférieure à celle de $V$ près. La troisième section est consacrée au cas où les valeurs de $\varphi$ sont seulement -1 et 1 , et au cas de la dimension deux. On termine dans la quatrième section par le cas où $S$ est basique.

\section{Spectre réel et formes quadratiques}

Pour une présentation du spectre réel on peut se référer à [6].

Soit $A$ un anneau commutatif unitaire. Le spectre réel de $A$, noté $\mathrm{Spec}_{r} A$, est l'ensemble des couples $(\mathfrak{p}, \alpha)$ où $\mathfrak{p}$ est un idéal premier de $A$ et $\alpha$ un ordre du corps résiduel $k(\mathfrak{p})=\operatorname{Frac}(A / \mathfrak{p})$. En particulier, si $A$ est un corps, alors $\operatorname{Spec}_{r} A$ est l'ensemble des ordres de $A$.

Si $\sigma=(\mathfrak{p}, \alpha)$ est un élément de $\operatorname{Spec}_{r} A$ et $f$ un élément de $A$, on écrit $f(\sigma)>0$ ou $f(\sigma)=1$ si l'image $\bar{f}$ de $f$ dans le corps résiduel $k(\mathfrak{p})$ est strictement positive pour $\alpha$. De même on écrit $f(\sigma)<0$ ou $f(\sigma)=-1$ si $\bar{f}$ est strictement négative pour $\alpha$, et $f(\sigma)=0$ si $\bar{f}=0$.

Un sous-ensemble constructible de $\operatorname{Spec}_{r} A$ est un ensemble de la forme

$$
\bigcup_{i=1}^{p}\left\{\sigma \in \operatorname{Spec}_{r} A \mid g_{i}(\sigma)=0, f_{i, 1}(\sigma)>0, \ldots, f_{i, r_{i}}(\sigma)>0\right\}
$$

où les $g_{i}$ et les $f_{i, j}$ sont des éléments de $A$. Les sous-ensembles constructibles forment

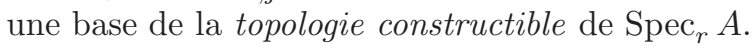

Une forme quadratique de dimension $r$ sur $\operatorname{Spec}_{r} A$ est la classe d'un $r$-uplet d'éléments de $A$ modulo la relation

$$
\left(f_{1}, \ldots, f_{r}\right) \sim\left(g_{1}, \ldots, g_{r}\right) \quad \Leftrightarrow \quad \forall \sigma \in \operatorname{Spec}_{r} A, \quad \sum_{i=1}^{r} f_{i}(\sigma)=\sum_{i=1}^{r} g_{i}(\sigma) .
$$


On note $\left\langle f_{1}, \ldots, f_{r}\right\rangle$ la classe de $\left(f_{1}, \ldots, f_{r}\right)$. La signature de la forme $\rho=\left\langle f_{1}, \ldots, f_{r}\right\rangle$ est la fonction $\widehat{\rho}: \operatorname{Spec}_{r} A \rightarrow \mathbb{Z}$ définie par $\widehat{\rho}(\sigma)=\sum_{i=1}^{r} f_{i}(\sigma)$. Pour les formes quadratiques sur le spectre réel, on peut se référer à [3] ou [12].

Dans [5], E. Becker et L. Bröcker ont donné la caractérisation suivante des signa-

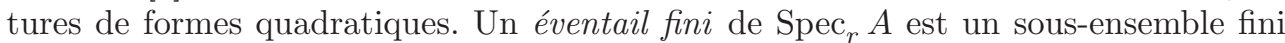
non vide $F$ de $\operatorname{Spec}_{r} A$ de la forme $\left\{\left(\mathfrak{p}, \alpha_{1}\right), \ldots,\left(\mathfrak{p}, \alpha_{s}\right)\right\}$ tel que pour tous $i_{1}, i_{2}, i_{3} \in$ $\{1, \ldots, s\}$, le produit $\alpha=\alpha_{i_{1}} \alpha_{i_{2}} \alpha_{i_{3}}$ soit un ordre de $k(\mathfrak{p})$ et $(\mathfrak{p}, \alpha)$ soit un élément de $F$. (Le produit des ordres signifie le produit des signes pour ces ordres).

Théorème 1.1 (de Représentation). Soit $\varphi: \operatorname{Spec}_{r} A \rightarrow \mathbb{Z}$ une fonction continue pour la topologie constructible de $\mathrm{Spec}_{r}$ A et la topologie discrète de $\mathbb{Z}$. La fonction $\varphi$ est une signature de forme quadratique sur $\operatorname{Spec}_{r} A$ si et seulement si pour tout éventail fini $F$ de $\operatorname{Spec}_{r} A$, on a

$$
\sum_{\sigma \in F} \varphi(\sigma) \equiv 0 \bmod |F| .
$$

Supposons que $A$ soit un corps $K$. Tout sous-ensemble de $\operatorname{Spec}_{r} K$ à un ou deux éléments est un éventail, appelé éventail trivial. Soit $B$ un anneau de valuation de $K$ et $\sigma$ un ordre de $K$. On dit que $B$ et $\sigma$ sont compatibles si pour tout $f \in B$ et tout $g$ dans l'idéal maximal $\mathfrak{m}$ de $B$, la relation $0<f<g$ pour $\sigma$ implique $f \in \mathfrak{m}$. Dans ce cas, $\sigma$ induit un ordre unique $\bar{\sigma}$ sur le corps résiduel $k$ de $B$, défini par $\bar{\sigma}(\bar{f})=\sigma(f)$ si $f \in B \backslash \mathfrak{m}$ et si $\bar{f}$ désigne la classe de $f$ dans $k$. Si $F$ est un éventail de $K$ dont tous les ordres sont compatibles avec $B$, alors les ordres de $k$ induits par les éléments de $F$ forment un éventail de $k$.

On a le théorème suivant, cf. [3, Th. VI.1.6].

Théorème 1.2 (de Trivialisation). Soit $K$ un corps. Pour tout éventail $F$ de $K$, il existe un anneau de valuation $B$ de $K$ compatible avec $F$ et tel que l'éventail induit par $F$ sur le corps résiduel de B soit trivial.

Inversement, si $B$ est un anneau de valuation de $K$, et si $\bar{\sigma}$ est un ordre du corps résiduel $k$ de $B$, les ordres de $K$ compatibles avec $B$ et induisant $\bar{\sigma}$ sont appelés relèvements de $\bar{\sigma}$ via $B$. Si $\bar{F}$ est éventail de $k$, l'ensemble des relèvements des éléments de $\bar{F}$ via $B$ forme un éventail de $K$.

Par le Théorème de Baer-Krull ([6, Th. 10.1.10]), si $B$ est un anneau de valuation discrète de rang $d$, tout ordre de $k$ a exactement $2^{d}$ relèvements via $B$.

Exemple 1.3. Soit $A$ un anneau local régulier de dimension $d$, de corps des fractions $K$ et de corps résiduel $k$. Fixons un système régulier de paramètres $x_{1}, \ldots, x_{d}$ de $A$. On construit alors un anneau de valuation discrète $B$ de $K$, de rang $d$, dominant $A$, et de même corps résiduel $k$. On procède par récurrence sur $d$, en utilisant l'anneau $A /\left(x_{1}\right)$ qui est local régulier de dimension $d-1$, cf. [4, Ex. 2.2]. L'anneau $B$ est alors l'anneau de valuation de la place $K=K_{0} \rightarrow K_{1} \cup \infty \rightarrow \cdots \rightarrow K_{d} \cup \infty$, où $K_{i}$ est le corps des fractions de l'anneau $A_{i}=A /\left(x_{1}, \ldots, x_{i}\right)$, et où la place $K_{i} \rightarrow K_{i+1} \cup \infty$ 
correspond à l'anneau de valuation discrète $A_{i\left(\bar{x}_{i+1}\right)}$ de $K_{i}$. Si $\tau \in \operatorname{Spec}_{r} k$, chacun des $2^{d}$ relèvements de $\tau$ via $B$ est déterminé par les signes qu'il donne à $x_{1}, \ldots, x_{d}$.

Considérons maintenant un ensemble algébrique réel $V \subset \mathbb{R}^{N}$. Notons $\mathcal{P}(V)$ l'anneau des polynômes sur $V$ et $\mathcal{R}(V)$ l'anneau des fonctions régulières sur $V$. Si $V$ est irréductible, soit $\mathcal{K}(V)$ le corps des fractions rationnelles sur $V$.

L'application qui à un sous-ensemble semi-algébrique $S$ de $V$, associe le constructible $\widetilde{S}$ de $\operatorname{Spec}_{r} \mathcal{P}(V)$ défini par la même combinaison booléenne d'équations et d'inégalités polynomiales, est bien définie. Elle induit un isomorphisme entre l'algèbre

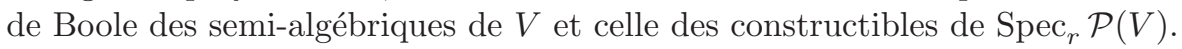

Soit $\varphi: S \rightarrow \mathbb{Z}$ une fonction constructible sur un semi-algébrique $S$ de $V$, donnée par la formule $\varphi=\sum_{i=1}^{r} m_{i} \mathbf{1}_{S_{i}}$ avec $m_{i} \in \mathbb{Z}$ et $S_{i} \subset S$ semi-algébrique. Elle induit une fonction $\widetilde{\varphi}: \widetilde{S} \rightarrow \mathbb{Z}$, définie par $\widetilde{\varphi}=\sum_{i=1}^{r} m_{i} \mathbf{1}_{\widetilde{S_{i}}}$.

Si $S=V$, la fonction $\varphi$ est algébriquement constructible si et seulement si $\tilde{\varphi}$ est une signature de formes quadratiques : " $\varphi=\sum_{i=1}^{r} \operatorname{sgn} P_{i}$ sur $V$ " équivaut à " $\widetilde{\varphi}$ signature de la forme $\left\langle P_{1}, \ldots, P_{r}\right\rangle$ sur $\operatorname{Spec}_{r} \mathcal{P}(V)$ ".

On dit que $\varphi$ est génériquement algébriquement constructible s'il existe $\psi: V \rightarrow \mathbb{Z}$ algébriquement constructible, et $W \subset V$ sous-ensemble algébrique de codimension au moins un, tels que $\varphi_{\mid V \backslash W}=\psi_{\mid V \backslash W}$. Si $V$ est irréductible, cela équivaut à ce que la restriction de $\widetilde{\varphi}$ à $\operatorname{Spec}_{r} \mathcal{K}(V)$ soit la signature d'une forme quadratique sur $\operatorname{Spec}_{r} \mathcal{K}(V)$.

Le Théorème de Représentation se reformule donc ainsi :

Proposition 1.4. Soit $V \subset \mathbb{R}^{N}$ un ensemble algébrique réel et soit $\varphi: V \rightarrow \mathbb{Z}$ une fonction constructible.

La fonction $\varphi$ est algébriquement constructible sur $V$ si et seulement si pour tout éventail fini $F$ de $\operatorname{Spec}_{r} \mathcal{P}(V)$, on a

$$
\sum_{\sigma \in F} \widetilde{\varphi}(\sigma) \equiv 0 \bmod |F|
$$

Si $V$ est irréductible, elle est génériquement algébriquement constructible sur $V$ si et seulement si la même relation est vérifiée pour tout éventail fini $F$ de $\operatorname{Spec}_{r} \mathcal{K}(V)$.

\section{Réduction à un problème générique}

Soient $V \subset \mathbb{R}^{N}$ un ensemble algébrique réel, $S$ un sous-ensemble semi-algébrique de $V$ et $\varphi$ une fonction constructible sur $S$.

Commençons par étudier le cas de la dimension un. Si $\operatorname{dim} V=1$, alors les fonctions algébriquement constructibles sur $V$ sont les fonctions constructibles sur $V$ qui sont constantes modulo 2, sauf en un nombre fini de points, sur chaque composante irréductible de $V$. Donc $\varphi$ est la restriction à $S$ d'une fonction algébriquement constructible sur $V$ si et seulement si pour toute composante irréductible $V^{\prime}$ de $V$, la fonction $\varphi$ est constante modulo 2 sur $S \cap V^{\prime}$ sauf en un nombre fini de points. 
Nous allons montrer comment, à l'aide d'une récurrence sur la dimension, on peut se ramener à l'étude du problème générique.

Proposition 2.1. Soit $V \subset \mathbb{R}^{N}$ un ensemble algébrique réel, $S \subset V$ un sous-ensemble semi-algébrique, et $\varphi: S \rightarrow \mathbb{Z}$ une fonction constructible. Notons

$$
X=\operatorname{Sing}(V) \cup \bigcup_{n \in \mathbb{Z}} \partial_{Z}\left(\varphi^{-1}(n)\right)
$$

où $\partial_{Z} T$ désigne la frontière de Zariski du semi-algébrique T, i.e. la clôture de Zariski de la frontière euclidienne de T. L'union est en fait finie.

La fonction $\varphi$ est la restriction à $S$ d'une fonction algébriquement constructible sur $V$ si et seulement si $\varphi$ est la restriction à $S$ d'une fonction génériquement algébriquement constructible sur $V$ et $\varphi_{\mid X \cap S}$ est la restriction à $X \cap S$ d'une fonction algébriquement constructible sur $X$.

Remarquons que $X$ est un ensemble algébrique tel que $\operatorname{dim} X<\operatorname{dim} V$. La proposition 2.1, avec une récurrence sur la dimension, permet donc de réduire notre problème à un nombre fini de problèmes similaires mais génériques, ainsi qu'à un problème similaire en dimension un (que l'on sait résoudre d'après le début de la section).

Démonstration de la Proposition 2.1. La condition est clairement nécessaire. Montrons qu'elle est aussi suffisante. Soit $\varphi^{\prime}: V \rightarrow \mathbb{Z}$ une fonction algébriquement constructible, et soit $Y \subset V$ un ensemble algébrique, tels que $\varphi_{\mid S \backslash Y}=\varphi_{\mid S \backslash Y}^{\prime}$ et $\operatorname{dim} Y<\operatorname{dim} V$. Soit $\varphi^{\prime \prime}: X \rightarrow \mathbb{Z}$ une fonction algébriquement constructible sur $X$ telle que $\varphi_{\mid S \cap X}=\varphi_{\mid S \cap X}^{\prime \prime}$.

Si $Y \subset X$, on définit $\psi: V \rightarrow \mathbb{Z}$ par $\psi(x)=\varphi^{\prime}(x)$ si $x \in V \backslash X$ et $\psi(x)=$ $\varphi^{\prime \prime}(x)$ si $x \in X$. La fonction $\psi$ ainsi construite est algébriquement constructible, et sa restriction à $S$ est égale à $\varphi$.

Si maintenant $Y$ n'est pas contenu dans $X$, soit $Y_{0}$ une composante irréductible de $Y$ qui n'est pas contenue dans $X$. Il existe une composante irréductible $V_{0}$ de $V$ qui contient $Y_{0}$. Comme $Y_{0}$ n'est pas contenu dans le lieu singulier de $V_{0}$, l'anneau $\mathcal{R}\left(V_{0}\right)_{\mathcal{I}\left(Y_{0}\right)}$ est local régulier de dimension $d$, où $d$ est la codimension de $Y_{0}$ dans $V_{0}$. Soit $x_{1}, \ldots, x_{d}$ un système régulier de paramètres de $\mathcal{R}\left(V_{0}\right)_{\mathcal{I}\left(Y_{0}\right)}$. Notons $C$ l'anneau de valuation discrète de rang $d$, dominant $\mathcal{R}\left(V_{0}\right)_{\mathcal{I}\left(Y_{0}\right)}$, construit à l'aide du système $x_{1}, \ldots, x_{d}$ comme dans l'exemple 1.3 .

On définit $\theta: \operatorname{Spec}_{r} \mathcal{K}\left(Y_{0}\right) \rightarrow \mathbb{Z}$ de la manière suivante. Soit $\bar{\sigma}$ un élément de $\operatorname{Spec}_{r} \mathcal{K}\left(Y_{0}\right)$, et notons $F_{\bar{\sigma}}$ l'ensemble des $2^{d}$ relèvements de $\bar{\sigma} \operatorname{dans~}_{\operatorname{Spec}_{r}} \mathcal{K}(V)$ via $C$. On pose $\theta(\bar{\sigma})=\frac{1}{2^{d}} \sum_{\sigma \in F_{\bar{\sigma}}} \widetilde{\varphi}^{\prime}(\sigma)$. Remarquons que $\theta(\bar{\sigma}) \in \mathbb{Z}$, car $F_{\bar{\sigma}}$ est un éventail de $\operatorname{Spec}_{r} \mathcal{K}\left(V_{0}\right)$ et $\varphi^{\prime}$ est algébriquement constructible. On obtient une application

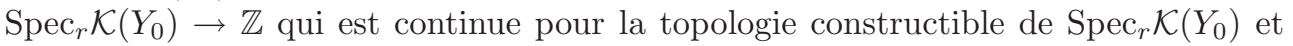
pour la topologie discrète de $\mathbb{Z}$. 
Mieux, la fonction $\theta$ est une signature de forme sur $\operatorname{Spec}_{r} \mathcal{K}\left(Y_{0}\right)$. En effet, soit $\bar{F}$ un éventail du corps $\mathcal{K}\left(Y_{0}\right)$. Notons $F$ son relèvement dans $\mathcal{K}(V)$ via $C$. On a

$$
\sum_{\bar{\sigma} \in \bar{F}} \theta(\bar{\sigma})=\frac{1}{2^{d}} \sum_{\sigma \in F} \widetilde{\varphi}^{\prime}(\sigma) \equiv 0 \quad \bmod \frac{|F|}{2^{d}}=|\bar{F}| .
$$

De plus, si $\bar{\sigma} \in \widetilde{S} \cap \operatorname{Spec}_{r} \mathcal{K}\left(Y_{0}\right)$, alors $\bar{\sigma}$ est dans $\left.\overline{\operatorname{Int}} \widetilde{\left(\varphi^{-1}(n)\right.}\right)$ pour un certain $n \in \mathbb{Z}$, puisque $Y_{0} \not \subset \bigcup \partial_{Z} \varphi^{-1}(n)$. Tous les éléments de $F_{\bar{\sigma}}$ sont alors aussi dans $\left.\operatorname{Int} \widetilde{\left(\varphi^{-1}(n)\right.}\right)$, donc $\theta(\bar{\sigma})=n=\widetilde{\varphi}(\bar{\sigma})$. Ainsi $\theta$ prolonge $\widetilde{\varphi}_{\mid \operatorname{Spec}_{r} \mathcal{K}\left(Y_{0}\right) \cap \widetilde{S}}$.

Soit $\varphi_{0}$ une fonction algébriquement constructible sur $Y_{0}$ telle que $\varphi_{0 \mid \operatorname{Spec}_{r}} \mathcal{K}\left(Y_{0}\right)=$ $\theta$. Elle coïncide donc avec $\varphi$ sur $\left(Y_{0} \backslash Z\right) \cap S$, avec $Z$ un sous-ensemble algébrique de $Y_{0}$ tel que $\operatorname{dim} Z<\operatorname{dim} Y_{0}$. On définit $\psi: V \rightarrow \mathbb{Z}$ par $\psi(x)=\varphi^{\prime}(x)$ si $x \in V \backslash Y_{0}$ et $\psi(x)=\varphi_{0}(x)$ si $x \in Y_{0}$. On obtient une fonction algébriquement constructible sur $V$ qui coïncide avec $\varphi$ sur $\left(V \backslash Y^{\prime}\right) \cap S$, où $Y^{\prime}$ est la réunion de $Z$ et des composantes irréductibles de $Y$ différentes de $Y_{0}$.

En répétant ce raisonnement sur les composantes de $Y^{\prime}$ non contenues dans $X$, et en effectuant une récurrence sur la dimension de ces composantes, on se ramène au cas où $Y \subset X$.

A partir de maintenant, on essaie donc de déterminer si $\varphi$ est restriction d'une fonction génériquement algébriquement constructible. En particulier, on supposera que le semi-algébrique $S$ est de même dimension que $V$.

On peut encore faire les simplifications suivantes à notre problème.

Une fonction sur $V$ est algébriquement constructible si et seulement si sa restriction à chaque composante irréductible de $V$ est algébriquement constructible. On peut donc supposer que $V$ est irréductible.

Si $V$ est non compact, soit $\bar{V}$ sa compactification à un point. Soit $\psi$ une fonction constructible sur $V$, que l'on prolonge en une fonction $\bar{\psi}$ sur $\bar{V}$ en donnant n'importe quelle valeur entière au point. Alors $\psi$ est algébriquement constructible sur $V$ si et seulement si $\bar{\psi}$ est algébriquement constructible sur $\bar{V}$. On peut donc aussi se restreindre au cas où $V$ est compact.

Si $\pi: V^{\prime} \rightarrow V$ est une suite d'éclatements à centres lisses, alors une fonction constructible $\psi: V \rightarrow \mathbb{Z}$ est génériquement algébriquement constructible sur $V$ si et seulement si $\psi \circ \pi$ l'est sur $V^{\prime}$. Quitte à faire des éclatements pour désingulariser $V$, on peut donc supposer de plus que $V$ est non singulier.

Définition 2.2. Soit $S$ un sous-ensemble semi-algébrique d'un ensemble algébrique non singulier $V$. Les murs de $S$ sont les composantes irréductibles de codimension 1 dans $V$ de $\partial_{Z}\left(S^{*}\right)$, où $S^{*}=\operatorname{Int}(\operatorname{Adh}(S))$.

Si $\varphi: S \rightarrow \mathbb{Z}$ est une fonction constructible sur $S$, les murs de $\varphi$ sont les murs des $\varphi^{-1}(n)$ pour $n \in \mathbb{Z}$. En particulier, les murs de $S$ sont des murs de $\varphi$. 
Soit $T$ une composante connexe du complémentaire dans $V$ de la réunion des murs de $\varphi$. Alors, ou bien $T$ est génériquement contenue dans le complémentaire de $S$ dans $V$, ou bien $T$ est génériquement contenue dans $S$ et $\varphi$ est génériquement constante sur $T$.

La notion de mur a été utilisée pour étudier la basicité des semi-algébriques ([2]), leur séparabilité ([1]), ainsi que pour caractériser les fonctions algébriquement constructibles $([7])$ et pour déterminer le nombre minimal de polynômes nécessaire à la description d'une fonction algébriquement constructible donnée ([8]).

Quitte à faire des éclatements supplémentaires, on peut supposer que les murs de $\varphi$ sont non singuliers et à croisements normaux.

\section{Cas où -1 et 1 sont seules valeurs}

Soit $V \subset \mathbb{R}^{N}$ un ensemble algébrique réel irréductible. Pour l'instant on ne fait pas d'hypothèse sur la dimension de $V$. Soient $S \subset V$ un semi-algébrique de même dimension que $V$ et $\varphi: S \rightarrow \mathbb{Z}$ une fonction constructible.

Une fonction algébriquement constructible sur un ensemble algébrique irréductible est génériquement constante modulo 2, car le signe d'un polynôme l'est. Une condition nécessaire pour que $\varphi$ soit la restriction à $S$ d'une fonction génériquement algébriquement constructible sur $V$ est donc que $\varphi$ soit génériquement constante modulo deux sur $S$. On fait désormais l'hypothèse que cette condition est vérifiée.

Lemme 3.1. Écrivons $\varphi=\varphi_{1}+\varphi_{2}$, où $\varphi_{1}, \varphi_{2}: S \rightarrow \mathbb{Z}$ sont deux fonctions constructibles telles que $\varphi_{1}$ soit génériquement constante modulo 4 et $\varphi_{2}$ ne prenne que les valeurs 1 et -1 . Alors $\varphi$ est la restriction à $S$ d'une fonction génériquement algébriquement constructible sur $V$ si et seulement si $\varphi_{1}$ et $\varphi_{2}$ le sont.

De plus, si $\varphi_{2}$ est restriction d'une fonction génériquement algébriquement constructible $\psi_{2}$ sur $V$, on peut supposer que $\psi_{2}$ est génériquement le signe d'un polynôme.

Preuve. La condition suffisante est claire. Pour la condition nécessaire, considérons une fonction algébriquement constructible $\psi: V \rightarrow \mathbb{Z}$ telle que $\psi_{\mid S}$ soit génériquement égale à $\varphi$, et écrivons $\psi=\operatorname{sgn} P_{1}+\cdots+\operatorname{sgn} P_{r}$ avec $P_{1}, \ldots, P_{r} \in \mathcal{P}(V)$. Notons $P=P_{1} \cdots P_{r}$. Alors la fonction $\psi-\operatorname{sgn} P$ vaut génériquement $r-1$ modulo 4 . En particulier, $\varphi_{2}-(\operatorname{sgn} P)_{\mid S}$ est génériquement constante modulo 4 , donc $\varphi_{2}=(\operatorname{sgn} P)_{\mid S}$ ou $\varphi_{2}=-(\operatorname{sgn} P)_{\mid S}$.

Ainsi $\varphi_{2}$ est restriction du signe du polynôme $Q= \pm P$, et $\varphi_{1}$ est restriction de la fonction algébriquement constructible $\psi-\operatorname{sgn} Q$.

Remarque 3.2. Notons $d=\operatorname{dim} V$. Toute fonction constructible sur $V$ et divisible par $2^{d}$ est algébriquement constructible. Cela vient du fait que tout sous-ensemble semi-algébrique ouvert basique de $V$ peut être décrit par une conjonction de $d$ inégalités strictes, cf. [10, Th. 2.8].

En particulier, si $\varphi$ est génériquement constante modulo $2^{d}$ sur $S$, alors elle est restriction d'une fonction génériquement algébriquement constructible sur $V$. On peut 
donc se ramener au cas où les seules valeurs génériques de $\varphi$ sont $-2^{d-1}+1,-2^{d-1}+$ $3, \ldots, 2^{d-1}-3,2^{d-1}-1$.

Le Lemme 3.1 justifie que l'on s'intéresse au prolongement en fonctions algébriquement constructibles de fonctions constructibles $S \rightarrow\{-1,1\}$. Par la Remarque 3.2, si $V$ est de dimension deux il suffit de considérer ce cas-là.

Définition 3.3. Deux sous-ensembles semi-algébriques $A, B$ de $V$ sont dits génériquement séparables s'il existe un polynôme $P \in \mathcal{P}(V) \backslash\{0\}$ vérifiant $P_{\mid A} \geq 0$ et $P_{\mid B} \leq 0$.

Le Lemme 3.1 donne immédiatement le résultat suivant :

Lemme 3.4. Si les seules valeurs de $\varphi$ sont -1 et 1 , alors $\varphi$ est restriction d'une fonction génériquement algébriquement constructible sur $V$ si et seulement si $\varphi^{-1}(1)$ et $\varphi^{-1}(-1)$ sont génériquement séparables.

On va utiliser les résultats de [1] sur la séparation des ensembles semi-algébriques pour étudier notre problème.

Si $W$ est un mur de $\varphi$, on définit une fonction $\partial_{W} \varphi \operatorname{sur} \operatorname{Adh}\left(S^{*}\right) \cap W \operatorname{par} \partial_{W} \varphi(x)=$ $\frac{1}{\left|I_{x}\right|} \sum_{n \in I_{x}} n$ avec $I_{x}=\left\{n \in \mathbb{Z} \mid x \in \operatorname{Adh}\left(\varphi^{-1}(n)^{*}\right)\right\}$. Si $V$ est non singulier, alors pour un point générique $x \operatorname{de} \operatorname{Adh}\left(S^{*}\right) \cap W$, l'ensemble $I_{x}$ a un ou deux éléments, et la valeur $\partial_{W} \varphi(x)$ est entière puisque $\varphi$ est génériquement constante modulo 2 .

Théorème 3.5. Soit $V \subset \mathbb{R}^{N}$ un ensemble algébrique réel irréductible, compact et non singulier. Soit $S$ un sous-ensemble semi-algébrique de $V$, de même dimension que $V$. On considère une fonction constructible $\varphi: S \rightarrow\{-1,1\}$, à murs non singuliers et à croisements normaux.

La fonction $\varphi$ est la restriction à $S$ d'une fonction génériquement algébriquement constructible sur $V$ si et seulement si pour tout mur $W$ de $V$,

(i) ou bien $\partial_{W} \varphi$ est la restriction à $\operatorname{Adh}\left(S^{*}\right) \cap W$ d'une fonction génériquement algébriquement constructible sur $W$, mais n'est pas génériquement nulle

(ii) ou bien, en fixant un paramètre uniformisant $t \in \mathcal{P}(V)$ de l'anneau $\mathcal{R}(V)_{\mathcal{I}(W)}$ et en notant $\varphi_{t}=(\operatorname{sgn} t) \cdot \varphi$, la fonction $\partial_{W} \varphi_{t}$ est la restriction à $\operatorname{Adh}\left(S^{*}\right) \cap W$ d'une fonction génériquement algébriquement constructible sur $W$, mais n'est pas génériquement nulle.

Par récurrence sur la dimension, on se ramène grâce à ce résultat à un nombre fini de problèmes de prolongement en dimension un, problèmes que l'on sait résoudre.

Preuve du Théorème 3.5. On utilise les notions d'ombre et contre-ombre, définies dans [1]. Si $W$ est un mur commun à deux semi-algébriques $A$ et $B$, l'ombre de $A$ dans $W$ est $\operatorname{Adh}(A) \cap W$ et celle de $B$ est $\operatorname{Adh}(B) \cap W$. La contre-ombre de $A$ dans $W$ est l'ombre de $(A \cap\{t>0\}) \cup(B \cap\{t<0\})$, et la contre-ombre de $B$ est l'ombre de 
$(A \cap\{t<0\}) \cup(B \cap\{t>0\})$, où $t$ désigne un paramètre uniformisant de l'anneau de valuation $\mathcal{R}(V)_{\mathcal{I}(W)}$.

Ici on travaille avec $\varphi^{-1}(1)^{*}$ et $\varphi^{-1}(-1)^{*}$. Si $W$ est un mur de $\varphi^{-1}(1)^{*}$ et $\varphi^{-1}(-1)^{*}$, l'ombre de $\varphi^{-1}(1)^{*}$ dans $W$ est génériquement égale à $\partial_{W} \varphi^{-1}(1) \cup \partial_{W} \varphi^{-1}(0)$, et sa contre-ombre à $\partial_{W} \varphi_{t}^{-1}(1) \cup \partial_{W} \varphi_{t}^{-1}(0)$. L'ombre de $\varphi^{-1}(-1)^{*}$ dans $W$ est génériquement égale à $\partial_{W} \varphi^{-1}(-1) \cup \partial_{W} \varphi^{-1}(0)$, et sa contre-ombre à $\partial_{W} \varphi_{t}^{-1}(-1) \cup \partial_{W} \varphi_{t}^{-1}(0)$.

Le Théorème 4.5 de [1] affirme que $\varphi^{-1}(1)$ et $\varphi^{-1}(-1)$ sont génériquement séparables dans $V$ si et seulement si pour chaque mur $W$ de $\varphi^{-1}(1)^{*}$ et $\varphi^{-1}(-1)^{*}$, ou bien leurs ombres, ou bien leurs contre-ombres, sont génériquement séparables dans $W$.

Or, par le Lemme 3.4, la séparabilité générique des semi-algébriques $\partial_{W} \varphi^{-1}(1) \cup$ $\partial_{W} \varphi^{-1}(0)$ et $\partial_{W} \varphi^{-1}(-1) \cup \partial_{W} \varphi^{-1}(0)$ équivaut au fait que les deux ensembles sont génériquement disjoints, et que la fonction $\partial_{W} \varphi$ est restriction d'une fonction génériquement algébriquement constructible sur $W$. De même pour les contre-ombres.

On conclut avec le Lemme 3.4.

Dans le cas où $V$ est de dimension deux, le Théorème 3.5 se simplifie.

Corollaire 3.6. Soit $V \subset \mathbb{R}^{N}$ un ensemble algébrique réel irréductible, compact et non singulier de dimension deux. Soient $S$ un sous-ensemble semi-algébrique de $V$ de dimension deux et $\varphi: S \rightarrow\{-1,1\}$ une fonction constructible, à murs non singuliers et à croisements normaux.

La fonction $\varphi$ est la restriction à $S$ d'une fonction génériquement algébriquement constructible sur $V$ si et seulement si pour tout mur $W$ de $\varphi$, la fonction $\partial_{W} \varphi$ est constante modulo 2 sur $S^{*} \cap W$ sauf en un nombre fini de points.

Preuve. Soit $W$ un mur, il est donc de dimension un. Supposons que $\partial_{W} \varphi$ est génériquement constante modulo 2 sur $S^{*} \cap W$. Si elle y est génériquement égale à 1 modulo 2, alors elle est génériquement constante modulo $2 \operatorname{sur} \operatorname{Adh}\left(S^{*}\right) \cap W$, donc $\partial_{W} \varphi$ est la restriction à $\operatorname{Adh}\left(S^{*}\right) \cap W$ d'une fonction algébriquement constructible sur $W$.

Si $\partial_{W} \varphi$ vaut génériquement 0 sur $S^{*} \cap W$, alors $\partial_{W} \varphi_{t}$ est génériquement égale à 1 modulo 2 sur $S^{*} \cap W$, et par l'argument précédent $\partial_{W} \varphi_{t}$ est la restriction à $\operatorname{Adh}\left(S^{*}\right) \cap W$ d'une fonction algébriquement constructible sur $W$. On conclut avec le Théorème 3.5 .

\section{Cas où $S$ est basique}

Soit $V$ un ensemble algébrique réel irréductible, et soit $S$ un sous-ensemble semialgébrique basique ouvert de $V$. Le sous-ensemble $X=\widetilde{S} \cap \operatorname{Spec}_{r} \mathcal{K}(V)$ forme ce

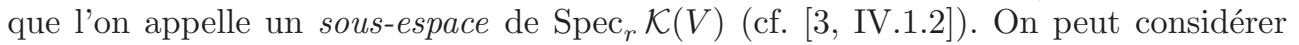
les formes quadratiques sur ce sous-espace. Une fonction à valeurs entières sur $X$, continue pour la topologie trace de $X$ et la topologie discrète de $\mathbb{Z}$, est la restriction à $X$ d'une signature de forme quadratique $\operatorname{sur} \operatorname{Spec}_{r} \mathcal{K}(V)$ si et seulement si c'est une 
signature de forme quadratique sur $X$. On a une caractérisation des signatures de formes quadratiques sur $X$ similaire au Théorème de Représentation (Théorème 1.1), en considérant les éventails de $\mathcal{K}(V)$ contenus dans $\widetilde{S}$.

Ces propriétés nous permettent de caractériser de manière effective les restrictions à $S$ de fonctions algébriquement constructibles.

Théorème 4.1. Soit $V \subset \mathbb{R}^{N}$ un ensemble algébrique réel irréductible, compact et non singulier. Soient $S$ un sous-ensemble semi-algébrique de $V$ et $\varphi: S \rightarrow \mathbb{Z}$ une fonction constructible. On suppose que $S$ est basique et non vide, et que les murs de $\varphi$ sont non singuliers et à croisements normaux.

La fonction $\varphi$ est la restriction à $S$ d'une fonction génériquement algébriquement constructible sur $V$ si et seulement si pour tout mur $W$ de $\varphi$, la fonction $\partial_{W} \varphi$ est la restriction à $\operatorname{Adh}\left(S^{*}\right) \cap W$ d'une fonction génériquement algébriquement constructible sur $W$.

Preuve. Supposons que $\varphi$ est la restriction à $S$ d'une fonction génériquement algébriquement constructible. Soit $W$ un mur de $\varphi$, et soit $\bar{F}$ un éventail de $\mathcal{K}(W)$ contenu dans $\operatorname{Adh} \widetilde{\left(S^{*}\right)} \cap W$. On considère l'éventail $F$ de $\mathcal{K}(V)$ formé des relèvements $\sigma$ d'éléments de $\bar{F}$ via $\mathcal{R}(V)_{\mathcal{I}(W)}$ tels que $\sigma \in \widetilde{S}$.

Premier CAS : $W$ est un mur de $S$. Comme $S$ est basique, par [1, Th. 4.1] la dimension de $S^{*} \cap W$ est strictement inférieure à la dimension de $W$. Chaque élément $\bar{\sigma}$ de $\bar{F}$ a exactement un relèvement $\sigma$ dans $F$, et $\widetilde{\partial_{W} \varphi}(\bar{\sigma})=\widetilde{\varphi}(\sigma)$. On a alors

$$
\sum_{\bar{\sigma} \in \bar{F}} \widetilde{\partial_{W} \varphi}(\bar{\sigma})=\sum_{\sigma \in F} \widetilde{\varphi}(\sigma) \equiv 0 \quad \bmod |F|=|\bar{F}|
$$

Deuxième CAS : $W$ n'est pas un mur de $S$. Chaque élément $\bar{\sigma}$ de $\bar{F}$ a exactement deux relèvements $\sigma$ et $\sigma^{\prime}$ dans $F$ et on a $\widetilde{\partial_{W} \varphi}(\bar{\sigma})=\frac{1}{2}\left(\widetilde{\varphi}(\sigma)+\widetilde{\varphi}\left(\sigma^{\prime}\right)\right)$. D'où

$$
\sum_{\bar{\sigma} \in \bar{F}} \widetilde{\partial_{W} \varphi}(\bar{\sigma})=\frac{1}{2} \sum_{\sigma \in F} \widetilde{\varphi}(\sigma) \equiv 0 \quad \bmod \frac{1}{2}|F|=|\bar{F}|
$$

Ainsi, par le Théorème de Représentation, $\partial_{W} \varphi$ est la restriction à $\operatorname{Adh}\left(S^{*}\right) \cap W$ d'une fonction génériquement algébriquement constructible sur $W$.

Inversement, supposons que $\varphi$ n'est pas la restriction à $S$ d'une fonction génériquement algébriquement constructible sur $V$. Par le début de la section, on sait qu'il existe un éventail $F$ de $\mathcal{K}(V)$, contenu dans $\widetilde{S}$, tel que $\sum_{\sigma \in F} \widetilde{\varphi}(\sigma) \not \equiv 0 \bmod |F|$. Choisissons $F$ de cardinal minimum pour cette propriété.

Par le Théorème de Trivialisation (Théorème 1.2), il existe un anneau de valuation $B$ de $\mathcal{K}(V)$ tel que $F$ et $B$ soient compatibles, et tel que $F$ induise au plus deux ordres $\tau_{1}^{\prime}, \tau_{2}^{\prime}$ sur le corps résiduel $k_{B}$ de $B$ (éventuellement, $\tau_{1}^{\prime}=\tau_{2}^{\prime}$ ). 
On a $\mathcal{R}(V) \subset B$ car $V$ est compact. L'intersection de l'idéal maximal de $B$ avec $\mathcal{R}(V)$ est un idéal premier $\mathfrak{p}$ de $\mathcal{R}(V)$. Notons $A=\mathcal{R}(V)_{\mathfrak{p}}$ et soit $Z$ l'ensemble des zéros de $\mathfrak{p}$ dans $V$. Le corps $\mathcal{K}(Z)=k(\mathfrak{p})$ est un sous-corps de $k_{B}$. Soient $\tau_{1}, \tau_{2}$ les restrictions à $\mathcal{K}(Z)$ de $\tau_{1}^{\prime}, \tau_{2}^{\prime}$ respectivement.

Soient $P_{1}, \ldots, P_{s}$ des polynômes sur $V$ décrivant les $\varphi^{-1}(n)^{*}$ pour $m \in \mathbb{Z}$. Comme les $P_{i}$ sont à croisements normaux, il existe un système régulier de paramètres $x_{1}, \ldots, x_{d}$ de $A$, tel que pour tout $i=1, \ldots, s$, le polynôme $P_{i}$ soit un monôme dans $A$ pour ce système, i.e. $P_{i}=u_{i} x_{1}^{m_{1, i}} \cdots x_{d}^{m_{d, i}}$ avec $u_{i}$ un inversible de $A$, et $m_{1, i}, \ldots, m_{d, i}$ des entiers.

Si $\sigma \in F$, alors $\varphi(\sigma)$ est déterminé par $k \in\{1,2\}$ tel que $\sigma$ induise $\tau_{k}$ et par les signes $x_{1}(\sigma), \ldots, x_{d}(\sigma)$. Mieux, tout élément $\sigma \in F$ est déterminé par $k \in\{1,2\}$ tel que $\sigma$ induise $\tau_{k}$ et par les signes $x_{1}(\sigma), \ldots, x_{d}(\sigma)$. Ceci est donné par le Lemme 1 de $[7]$; c'est une conséquence de la minimalité du cardinal de $F$.

Comme $\varphi$ est génériquement constante modulo 2, le cardinal de $F$ est au moins 4 . Il existe donc $j_{0} \in\{1, \ldots, d\}$ tel que tous les éléments de $F$ ne donnent pas le même signe à $x_{j_{0}}$.

Soit $C$ l'anneau de valuation discrète dominant $A$ et de même corps résiduel, construit à l'aide du système $x_{j_{0}}, \ldots, x_{d}, x_{1}, \ldots, x_{j_{0}-1}$ comme dans l'exemple 1.3. Notons $H$ l'éventail de $\mathcal{K}(V)$ relèvement de $\tau_{1}, \tau_{2}$ via $C$. On considère l'application $\Theta: F \rightarrow H$ qui à un élément $\sigma$ de $F$ se spécialisant en $\tau_{k}$, associe l'élément de $H$ se spécialisant en $\tau_{k}$ et ayant le même signe que $\sigma$ sur $x_{1}, \ldots, x_{d}$. Soit $G=\Theta(F)$. On peut vérifier facilement que $G$ est un éventail, de même cardinal que $F$ car $\Theta$ est injective. (L'application $\Theta$ est ce que l'on appelle un morphisme d'espace des ordres). De plus, pour tout $\sigma \in F$ on a $\varphi(\Theta(\sigma))=\varphi(\sigma)$, donc $\sum_{\sigma \in G} \widetilde{\varphi}(\sigma) \not \equiv 0 \bmod |G|$.

Soit $K=\operatorname{Frac}\left(A /\left(x_{j_{0}}\right)\right)$ et soit $\bar{G}$ l'éventail de $K$ induit par $G$. Le cardinal de $G$ est $|\bar{G}|$ ou $2|\bar{G}|$.

Premier CAS : $j_{0}$ correspond à un mur $W$ de $S$. Comme dans le premier cas de l'implication réciproque, tout élément $\bar{\sigma} \in \bar{G}$ a exactement un relèvement dans $G$, et on a $\widetilde{\partial_{W} \varphi}(\bar{\sigma})=\widetilde{\varphi}(\sigma)$. On a donc

$$
\sum_{\bar{\sigma} \in \bar{G}} \widetilde{\partial_{W} \varphi}(\bar{\sigma}) \not \equiv 0 \quad \bmod |\bar{G}|
$$

et $\partial_{W} \varphi$ ne se prolonge pas en une fonction génériquement algébriquement constructible sur $W$.

Deuxième CAS : $j_{0}$ ne correspond pas à un mur de $S$ et $|G|=2|\bar{G}|$. Alors $j_{0}$ correspond à un mur $W$ de $\varphi$, car sinon pour tout $\bar{\sigma} \in \bar{G}$, les deux relèvements de $\bar{\sigma}$ prendraient la même valeur pour $\widetilde{\varphi}$. L'éventail $G \cap\left\{x_{j_{0}}>0\right\}$ contredirait la minimalité du cardinal de $F$.

Pour tout $\bar{\sigma} \in \bar{G}$, on a $\widetilde{\partial_{W} \varphi}(\bar{\sigma})=\frac{1}{2}\left(\widetilde{\varphi}(\sigma)+\widetilde{\varphi}\left(\sigma^{\prime}\right)\right)$, où $\sigma$ et $\sigma^{\prime}$ sont les deux relèvements de $\bar{\sigma}$ dans $G$. On a donc

$$
\sum_{\bar{\sigma} \in \bar{G}} \widetilde{\partial_{W} \varphi}(\bar{\sigma})=\frac{1}{2} \sum_{\sigma \in G} \widetilde{\varphi}(\sigma) \not \equiv 0 \quad \bmod \frac{|G|}{2}=|\bar{G}|
$$


et $\partial_{W} \varphi$ n'est pas la restriction d'une fonction génériquement algébriquement constructible sur $W$.

Troisième CAS : $j_{0}$ ne correspond pas à un mur de $S$ et $|G|=|\bar{G}|$. Soit $G^{\prime}$ le relèvement de $\bar{G}$ via $A_{\left(x_{j_{0}}\right)}$. Comme $j_{0}$ ne correspond pas à un mur de $S$, on a bien $G^{\prime} \subset \widetilde{S}$. On note $G^{+}=G^{\prime} \cap\left\{x_{j_{0}}>0\right\}$ et $G^{-}=G^{\prime} \cap\left\{x_{j_{0}}<0\right\}$. On construit aussi l'éventail $G^{\prime \prime}$, relèvement via $A_{\left(x_{j_{0}}\right)}$ de l'éventail induit sur $K$ par $G \cap G^{-}$. On a alors

$$
\sum_{\sigma \in G} \widetilde{\varphi}(\sigma)=\sum_{\sigma \in G^{+}} \widetilde{\varphi}(\sigma)-\sum_{\sigma \in G^{\prime \prime}} \widetilde{\varphi}(\sigma)+2 \sum_{\sigma \in G^{\prime \prime} \cap G^{-}} \widetilde{\varphi}(\sigma)
$$

Or $G^{+}$et $G^{\prime \prime}$ ont même cardinal que $G$, et $G^{\prime \prime} \cap G^{-}$a un cardinal moitié. Par minimalité du cardinal de $G$, on a donc $2 \sum_{\sigma \in G^{\prime \prime} \cap G^{-}} \widetilde{\varphi}(\sigma) \equiv 0 \bmod |G|$. Ainsi

(i) ou bien $\sum_{\sigma \in G^{\prime \prime}} \widetilde{\varphi}(\sigma) \not \equiv 0 \bmod \left|G^{\prime \prime}\right|$, et on est ramené au deuxième cas,

(ii) ou bien $\sum_{\sigma \in G^{+}} \widetilde{\varphi}(\sigma) \not \equiv 0 \bmod \left|G^{+}\right|$. On recommence notre raisonnement avec $G^{+}$, en choisissant un nouvel indice $j_{1}$ tel que tous les éléments de $G^{+}$n'aient pas le même signe pour $x_{j_{1}}$. Remarquons que le nombre de tels indices a été diminué d'un en passant de $G$ à $G^{+}$. Notre raisonnement finira donc bien par s'arrêter.

Remarque 4.2. Si le semi-algébrique $S$ n'est pas basique, alors $X=\widetilde{S} \cap \operatorname{Spec}_{r} \mathcal{K}(V)$

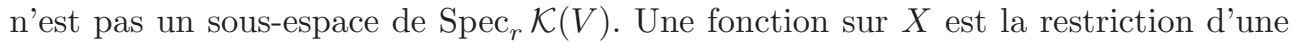
signature de forme quadratique $\operatorname{sur}^{\operatorname{Spec}_{r}} \mathcal{K}(V)$ si et seulement si c'est la restriction d'une signature de forme quadratique sur le sous-espace $X^{\prime}$ engendré par $X$.

Cependant, construire un prolongement de $\widetilde{\varphi}$ à $X^{\prime}$ n'est pas facile. En effet, on n'a pas unicité du prolongement, même si on restreint l'ensemble des valeurs prises par la fonction. Par exemple, la fonction suivante sur le semi-algébrique $S$ formé des deux cubes

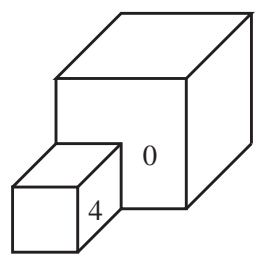

a parmi ses prolongements en fonctions algébriquement constructibles les deux fonctions suivantes, qui diffèrent sur $X^{\prime}$.
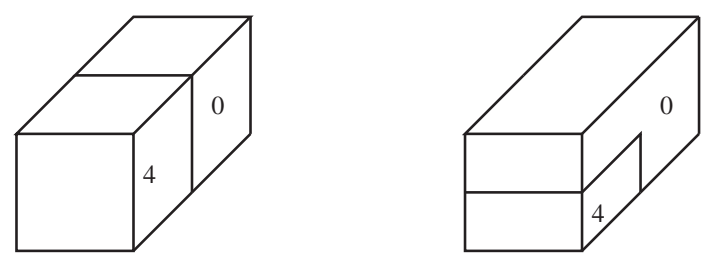
De plus, il n'existe pas toujours des prolongements n'ajoutant pas de murs. Par exemple, la fonction suivante est prolongeable en une fonction algébriquement constructible $\psi$ sur $\mathbb{R}^{2}$. (On peut prendre pour $\psi$ le signe de l'équation d'une droite verticale séparant les deux composantes connexes de $S$ ).

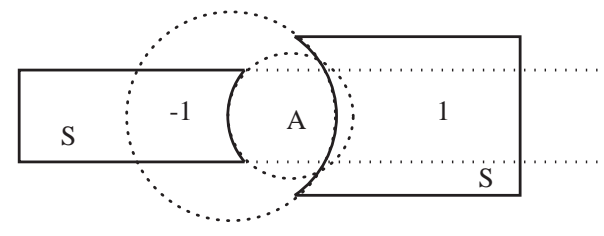

Cependant, $\psi$ aura plus de murs que $\varphi$. En effet, on peut supposer que $\psi$ est le signe d'un polynôme $P$. Ce polynôme ne doit changer de signe en traversant aucun des deux cercles, il ne peut donc pas être de signe constant sur la composante connexe $A$ du complémentaire de la réunion des murs de $\varphi$.

\section{Références}

[1] F. Acquistapace, C. Andradas, and F. Broglia, Separation of semialgebraic sets, J. Amer. Math. Soc. 12 (1999), 703-728.

[2] F. Acquistapace, F. Broglia, and M. P. Vélez, Basicness of semialgebraic sets, Geom. Dedicata 78 (1999), 229-240.

[3] C. Andradas, L. Bröcker, and J. M. Ruiz, Constructible sets in real geometry, Ergebnisse der Mathematik und ihrer Grenzgebiete (3), vol. 33, Springer-Verlag, Berlin, 1996, ISBN 3-54060451-0.

[4] C. Andradas and J. M. Ruiz, Low-dimensional sections of basic semialgebraic sets, Illinois J. Math. 38 (1994), 303-326.

[5] E. Becker and L. Bröcker, On the description of the reduced Witt ring, J. Algebra 52 (1978), 328-346.

[6] J. Bochnak, M. Coste, and M.-F. Roy, Real algebraic geometry, Ergebnisse der Mathematik und ihrer Grenzgebiete (3), vol. 36, Springer-Verlag, Berlin, 1998, ISBN 3-540-64663-9.

[7] I. Bonnard, Un critère pour reconnaître les fonctions algébriquement constructibles, J. Reine Angew. Math. 526 (2000), 61-88. (French, with English summary)

[8] _ Description of algebraically contructible functions, Adv. Geom. 3 (2003), 145-161.

[9] Michel Coste and Krzysztof Kurdyka, Le discriminant d'un morphisme de variétés algébriques réelles, Topology 37 (1998), 393-399.

[10] C. McCrory and A. Parusiński, Algebraically constructible functions, Ann. Sci. École Norm. Sup. (4) 30 (1997), 527-552. (English, with English and French summaries)

[11] $\ldots$ Topology of real algebraic sets of dimension 4 : necessary conditions, Topology 39 (2000), 495-523.

[12] M. A. Marshall, Spaces of orderings and abstract real spectra, Lecture Notes in Mathematics, vol. 1636, Springer-Verlag, Berlin, 1996, ISBN 3-540-61729-9.

[13] A. Parusiński and Z. Szafraniec, On the Euler characteristic of fibres of real polynomial maps, Singularities Symposium-Łojasiewicz 70 (Kraków, 1996 ; Warsaw, 1996), Banach Center Publ., vol. 44, Polish Acad. Sci., Warsaw, 1998, pp. 175-182. 